ORIGINAL ARTICLE

\title{
Experimental approaches to evaluate activities of cytochromes P450 3A
}

\author{
Lucie BOŘEK-DOHALSKÁ, Petr HODEK, Jiří HUDEČEK, Marie STIBOROVÁ
}

Department of Biochemistry, Faculty of Science, Charles University, Prague, Albertov 2030, 12840 Prague 2, CZECH REPUBLIC

ITX010208A05 • Received:2 July 2008 • Revised:8 August 2008 • Accepted: 18 August 2008 • Electronic Publication: November 2010

\begin{abstract}
Cytochrome P450 (CYP) is a heme protein oxidizing various xenobiotics, as well as endogenous substrates. Understanding which CYP enzymes are involved in metabolic activation and/or detoxication of different compounds is important in the assessment of an individual's susceptibility to the toxic action of these substances. Therefore, investigation which of several in vitro experimental models are appropriate to mimic metabolism of xenobiotics in organisms is the major challenge for research of many laboratories. The aim of this study was to evaluate the efficiency of different in vitro systems containing individual enzymes of the mixed-function monooxygenase system to oxidize two model substrates of CYP3A enzymes, exogenous and endogenous compounds, a-naphtoflavone (a-NF) and testosterone, respectively. Several different enzymatic systems containing CYP3A enzymes were utilized in the study: (i) human hepatic microsomes rich in CYP3A4, (ii) hepatic microsomes of rabbits treated with a CYP3A6 inducer, rifampicine, (iii) microsomes of Baculovirus transfected insect cells containing recombinant human CYP3A4 and NADPH:CYP reductase with or without cytochrome $b_{5}\left(\right.$ Supersomes $\left.{ }^{\top M}\right)$, (iv) membranes isolated from of Escherichia coli, containing recombinant human CYP3A4 and cytochrome $b_{5}$, and (v) purified human CYP3A4 or rabbit CYP3A6 reconstituted with NADPH:CYP reductase with or without cytochrome $b_{5}$ in liposomes. The most efficient systems oxidizing both compounds were Supersomes ${ }^{\text {TM }}$ containing human CYP3A4 and cytochrome $b_{5}$. The results presented in this study demonstrate the suitability of the supersomal CYP3A4 systems for studies investigating oxidation of testosterone and a-NF in vitro.
\end{abstract}

KEY WORDS: cytochrome P450 3A; a-naphthoflavone; metabolism

\section{INTRODUCTION}

The cytochrome P450s (CYP) are a family of hemoprotein enzymes that play important roles in the metabolism of drugs and carcinogens, as well as endogenous compounds such as prostaglandins, fatty acids and steroids (Gonzalez and Gelboin, 1992, Ortiz de Montellano 1995).

CYP3A is one of the major subfamilies expressed in human livers and is found at high levels in the intestinal tract (Hosea et al., 2000). This enzyme oxidizes endogenous and exogeneous compounds as well as over half of the drugs in therapeutic use (Hosea et al., 2000). The human CYP3A subfamily expressed in human livers consists of CYP3A4 (Beaune et al., 1986), CYP3A5 (Aoyama et al., 1989, Yamaori et al., 2003), and CYP3A7 (Kitada et al., 1985, Yamaori et al., 2003). CYP3A4 is the most abundant form of CYP3A ( 30\% of total CYP) expressed in adult human livers (Shimada et al.,

Correspondence address:

\section{Prof Marie Stiborová, DrSc.}

Department of Biochemistry, Faculty of Science, Charles University, Prague, Albertov 2030, 12840 Prague 2, Czech Republic

E-MAll: stiborov@natur.cuni.cz
1994). CYP3A enzymes are induced by rifampicin (RIF) in the human (CYP3A4/5) and rabbit (CYP3A6), but not in rat (CYP3A1/2). CYP3A4 demonstrates homotropic cooperativity (non-Michaelis-Menten kinetics) with a number of substrates (Atkins 2005, Ekins et al., 2003, Guengerich 1999, Tsalkova et al., 2007, Ueng et al., 1997). The enzyme is also known to exhibit heterotropic cooperativity, which is characterized by increased oxidation of one substrate in the presence of an effector, such as $\alpha$-naphthoflavone ( $\alpha$-NF), that may also serve as a substrate or inhibitor (Galetin et al., 2002, Harlow and Halpert 1998, Koley et al., 1997, Shou et al., 1994, Tang and Stearns 2001, Tsalkova et al., 2007). During the past decade understanding of the mechanism of CYP3A4 cooperativity has progressed from a static model with multiple binding sites (Domanski et al., 1998, Shou et al., 1994, Tsalkova et al., 2007, Ueng et al., 1997) to more complex dynamic model suggesting effector-induced conformational rearrangements of the enzyme along with multiple ligand binding (Atkins et al., 2001, Isin and Guengerich 2006).

CYP3A4 cooperativity may be influenced by the levels of the redox partners, such as cytochrome $b_{5}$ or cumene hydroperoxide, relative to the CYP (Kumar et al., 2005, Ueng et al., 1997). Cytochrome $b_{5}$ has been reported to stimulate CYP3A4, dependent on the specific substrate (Kumar et al., 2005, Patki et al., 2003, Yamazaki et al., 1999). 
The mode of action of cytochrome $b_{5}$ remains controversial (Kumar et al., 2005). Although the role of this protein as a source of electrons for CYPs is well known (Guryev et al., 2001, Schenkman and Jansson 1999, Yamazaki et al., 2001), increasing evidence points to an allosteric effects of cytochrome $b_{5}$ mediated in part by an effect on the CYP spin state (Reed and Hollenberg 2003a, Reed and Hollenberg 2003b). Its modulatory effect is further supported by the fact that cytochrome $b_{5}$ not only increases CYP activity but in some cases also inhibits its activity (Reed and Hollenberg 2003b, Yamaori et al., 2003). In addition, the interaction with cytochrome $b_{5}$ may affect the degree of oligomerization of CYP in membrane (Yamada et al., 1995).

Understanding which CYP enzymes are involved in the metabolic activation and/or detoxication of xenobiotics and endogenous compounds is important in the assessment of an individual's susceptibility to the toxic action of these substances. Therefore, investigation which of several in vitro experimental models are appropriate to mimic metabolism of xenobiotics in organisms is the major challenge for research of many laboratories.

The aim of the present work was to evaluate the efficiency of different in vitro systems containing individual enzymes of the mixed-function monooxygenase system to oxidize two model substrates of CYP3A, exogenous ( $\alpha-\mathrm{NF}$ ) and endogenous (testosterone) compounds, respectively.

\section{MATERIALS AND METHODS}

\section{Chemicals}

Glucose-6-phosphate, NADP+, NADPH, $\alpha-\mathrm{NF}, 3-[(3-\mathrm{chol}-$ amidopropyl)dimethyl-ammonio]-1-propane sulfonate (CHAPS), dilauroyl phosphatidylcholine, and dithiothreitol were obtained from Sigma Chemical Co. (St. Louis, MO, USA). Testosterone and 6 6 -hydroxytestosterone were purchased from Merck (Darmstadt, Germany). Glucose6-phosphate dehydrogenase was from Serva (Heidelberg, Germany). Bicinchoninic acid was from Pierce (Rockford, IL, USA). All chemicals were of a reagent grade or better.

\section{Animals and pretreatment}

Adult male rabbits $(2.5-3.0 \mathrm{~kg}$, VELAZ, The Czech Republic) were fed ad libitum on pellet chow and water one week before treatment. Then, rabbits were pretreated with RIF $(50 \mathrm{mg} / \mathrm{kg}$ in $40 \mathrm{mM} \mathrm{NaOH}$ i.p. for 3 consecutive days) and used for isolation of microsomes.

\section{Preparation of microsomes, isolation of enzymes and assays}

Microsomes were isolated from livers of rabbits pretreated with RIF as described previously (Stiborova et al., 1995, Stiborova et al., 1990) and stored in $0.5 \mathrm{ml}$ aliquots in liquid nitrogen until use. CYP3A6 was isolated from liver microsomes of rabbit induced by RIF. The procedure was analogous as described previously (Haugen and Coon 1976, Yang et al., 1985). Rabbit liver NADPH:CYP reductase was purified as described earlier (Yasukochi et al., 1979). Protein concentrations were assessed using the bicinchoninic acid protein assay with serum albumin as a standard
(Wiechelman et al., 1988). Total CYP content was measured based on complex of reduced CYP with CO (Omura and Sato 1964). Supersomes ${ }^{\mathrm{TM}}$ were from Gentest corp. (Woburn, MA). Membranes isolated from of Escherichia coli, containing human CYP3A4 was a gift from Dr. Soucek (National Institute of Public Health, Prague, Czech Republic). Purified human CYP3A4 was from Prof. Anzenbacher (Palacky University, Olomouc, Czech Republic) and human microsomes from Dr. Szotakova (Charles University, Hradec Kralove, Czech Republic).

\section{Testosterone $6 \beta$-hydroxylation}

The incubation mixtures for measuring the testosterone metabolism contained in a final volume of $0.5 \mathrm{ml}: 0.1 \mathrm{M}$ potassium phosphate buffer, $\mathrm{pH} 7.4,50 \mu \mathrm{M}$ testosterone ( $2 \mu \mathrm{l}$ of stock methanol solution per incubation), $10 \mathrm{mM}$ $\mathrm{MgCl}_{2}, 10 \mathrm{mM}$ D-glucose 6-phosphate, $1 \mathrm{mM} \mathrm{NADP}{ }^{+}$, $1 \mathrm{U} / \mathrm{ml} \mathrm{D}$-glucose 6-phosphate dehydrogenase and one from the used enzyme system: (i) human hepatic microsomes $(0.2 \mu \mathrm{M}$ CYP), (ii) hepatic microsomes of rabbits treated with a CYP3A6 inducer, rifampicine $(0.2 \mu \mathrm{M}$ CYP), (iii) microsomes of Baculovirus transfected insect cells containing recombinant human CYP3A4 $(0.05 \mu \mathrm{M})$ and NADPH:CYP reductase with or without cytochrome $b_{5}$ $(0.2 \mu \mathrm{M})$ (Supersomes $\left.{ }^{\mathrm{TM}}\right)$, (iv) membranes isolated from of Escherichia coli, containing recombinant human CYP3A4 $(0.05 \mu \mathrm{M})$ and cytochrome $b_{5}(0.2 \mu \mathrm{M})$ reconstituted with NADPH:CYP reductase $(0.05 \mu \mathrm{M})$, and (v) purified human CYP3A4 $(0.05 \mu \mathrm{M})$ or rabbit CYP3A6 $(0.2 \mu \mathrm{M})$ reconstituted with NADPH:CYP reductase $(0.05$ or $0.2 \mu \mathrm{M})$ with or without cytochrome $b_{5}(0.2$ or $0.8 \mu \mathrm{M})$ in liposomes. Microsomes and Supersomes ${ }^{\mathrm{TM}}$ were diluted on the concentration mentioned above. Bacterial membranes were reconstituted $10 \mathrm{~min}$ with $\mathrm{NADPH}$ :CYP reductase and cytochrome $b_{5}$ and then diluted with buffer on the used CYP concentration (see above). Reconstitution of purified CYP3A4 and CYP3A6 with NADPH:CYP reductase was carried out essentially as described earlier (Burke et al., 1985). Briefly, CYP3A were reconstituted as follows $(0.5 \mu \mathrm{M}$ CYP3A, $0.5 \mu \mathrm{M}$ NADPH:CYP reductase, $0.5 \mu \mathrm{g} / \mu \mathrm{l}$ CHAPS, $0.1 \mu \mathrm{g} / \mu \mathrm{l}$ vesicles (from D,L-dilauroylphosphatidylcholine), $3 \mathrm{mM}$ reduced glutathione and $50 \mathrm{mM}$ HEPES/KOH, $\mathrm{pH}$ 7.4). An aliquot containing $25 \mathrm{pmol}$ of reconstituted CYP3A4 or 100 pmol of reconstituted CYP3A6 was added to incubation mixtures. The mixtures were incubated for $15 \mathrm{~min}$, at $37^{\circ} \mathrm{C}$ in a shaking incubator. The reaction was terminated by addition of $0.1 \mathrm{ml}$ of $1 \mathrm{M}$ aqueous $\mathrm{Na}_{2} \mathrm{CO}_{3}$ containing $2 \mathrm{M} \mathrm{NaCl}$. Then, phenacetin ( $5 \mu \mathrm{l}$ of $1 \mathrm{mM}$ stock solution) was added as an internal standard. The metabolites were extracted with $2 \mathrm{ml}$ of $\mathrm{CH}_{2} \mathrm{Cl}_{2}$ and the extracts were evaporated to dryness. The residues were dissolved in the mobile phase for HPLC (see below).

\section{HPLC conditions}

Testosterone and its metabolites were separated on Nucleosil (C18) HPLC column $(4.6 \times 25 \mathrm{mM}, 5 \mu \mathrm{m}$, Macherey-Nagel, Germany). The flow rates, mobile phases and detection wavelengths for assays were $0.6 \mathrm{ml} / \mathrm{min}, 70: 30 \mathrm{CH}_{3} \mathrm{OH} / \mathrm{H}_{2} \mathrm{O}$ $(\mathrm{v} / \mathrm{v})$, and $254 \mathrm{~nm}$, respectively. 


\section{0xidation of a-NF}

Incubation mixtures contained in a final volume of $0.5 \mathrm{ml}$ : $0.1 \mathrm{M}$ potassium phosphate buffer, $\mathrm{pH} 7.4,150 \mu \mathrm{M} \alpha-\mathrm{NF}$ ( $2 \mu \mathrm{l}$ of stock methanol solution per incubation), $50 \mu \mathrm{l}$ of NADPH-generating system (see above) and the same enzyme systems as in the case of testosterone oxidation (see above). The mixtures were incubated for $30 \mathrm{~min}$ at $37^{\circ} \mathrm{C}$ in a shaking incubator. The reaction was terminated by addition of $0.1 \mathrm{ml}$ of $1 \mathrm{M}$ aqueous $\mathrm{Na}_{2} \mathrm{CO}_{3}$ containing $2 \mathrm{M} \mathrm{NaCl}$. The $\alpha$-NF metabolites were extracted with $2 \mathrm{ml}$ of $\mathrm{CH}_{2} \mathrm{Cl}_{2}$ and the extracts were evaporated to dryness. The residues were dissolved in the mobile phase for HPLC. Samples were analyzed by HPLC as described elsewhere (Hosea et al., 2000, Thakker et al., 1981) to identify a-NF oxidation products. Two metabolites with retention times of 13.0 and $21.0 \mathrm{~min}$ (Figure 2), which were previously assigned as the trans-7,8dihydrodiol and 5,6-epoxide were formed (Andries et al., 1990, Hosea et al., 2000, Shou et al., 1994). Mass spectroscopy (MALDI-TOF using $\alpha$-cyano-4-hydroxycinnamic acid as a matrix) of the metabolite with a retention time of $13.0 \mathrm{~min}$ gave molecular ions at $\mathrm{m} / \mathrm{z} 307(\mathrm{M}+\mathrm{H})^{+}$and $\mathrm{m} / \mathrm{z} 329(\mathrm{M}+\mathrm{Na})^{+}$, suggesting a dihydrodiol derivative. The metabolite with a retention time of $21.0 \mathrm{~min}$ gave molecular ions at $\mathrm{m} / \mathrm{z} 289(\mathrm{M}+\mathrm{H})^{+}$and $\mathrm{m} / \mathrm{z} 311(\mathrm{M}+\mathrm{Na})^{+}$and peaks at $\mathrm{m} / \mathrm{z} 273(\mathrm{M}+\mathrm{H})^{+}$and at $\mathrm{m} / \mathrm{z} 295(\mathrm{M}+\mathrm{Na})^{+}$, that is an indicative of an epoxide metabolite. The results are consistent with a previous studies on the metabolism of $\alpha-\mathrm{NF}$ by rat microsomes pretreated with 3-methylcholanthere (Andries et al., 1990) and by purified reconstituted CYP3A4 (Shou et al. 1994), in which these two metabolites were identified as trans-7,8-dihydrodiol (retention time $=13.0 \mathrm{~min}$ ) and 5,6 -epoxide (retention time $=21.0 \mathrm{~min}$ ). Another minor metabolite eluted with retention time of $9.6 \mathrm{~min}$ has not been identified yet (Figure 2). Two peaks eluted with retention times of 26.6 and 28.2 min seem not to be the products of $\alpha-\mathrm{NF}$ oxidation, because they are also present in the control reaction samples. These samples containing all reaction components were immediately (without incubation) applied on a HPLC column to analyze.

\section{RESULTS}

Several different systems containing CYP3A enzymes were utilized to investigate oxidation of testosterone and $\alpha-\mathrm{NF}$ in vitro: (i) human hepatic microsomes rich in CYP3A4, (ii) hepatic microsomes of rabbit treated with CYP3A6 inducer, rifampicine, (iii) microsomes of Baculovirus transfected insect cells containing recombinant human CYP3A4 and NADPH:CYP reductase with or without cytochrome $b_{5}$ $\left(\right.$ Supersomes $\left.{ }^{\mathrm{Tu}}\right)$, (iv) membranes isolated from of Escherichia coli, containing human CYP3A4 with or without cytochrome $\mathrm{b}_{5}$, and (v) purified human CYP3A4 or rabbit CYP3A6 reconstituted with NADPH:CYP reductase with or without cytochrome $b_{5}$ in liposomes.

The most efficient systems oxidizing testosterone to its $6 \beta$-hydroxylated metabolite were Supersomes ${ }^{\mathrm{Tm}}$ containing human CYP3A4 and cy tochrome $b_{5}$ (Figure 1), while low efficiency of CYP3A4 and/or CYP3A6 expressed in membranes of $E$. coli or reconstituted with NADPH:CYP reductase in liposomes were found (Figure 1). The activity of purified CYP3A4 or CYP3A6 reconstituted with NADPH:CYP reductase (measured as testosterone $6 \beta$-hydroxylation) was enhanced by cytochrome $b_{5}$. This protein did not influence oxidation of testosterone by recombinant CYP3A4 of E.coli membranes.

$\alpha-\mathrm{NF}$ was oxidized into two metabolites, tentatively identified as trans-7,8-dihydrodiol (retention time $=13.0 \mathrm{~min}$ ) and 5,6-epoxide (retention time $=21.0 \mathrm{~min}$ ) by mass spectra and previously reported data (Andries et al., 1990, Shou et al., 1994) (Figure 2). Under the condition used, 5,6-epoxide was the major product in all systems used. The most efficient system oxidizing $\alpha-\mathrm{NF}$ was Supersomes ${ }^{\mathrm{Tm}}$ containing human CYP3A4 and cytochrome $b_{5}$ (Figure 3). Also low efficiency

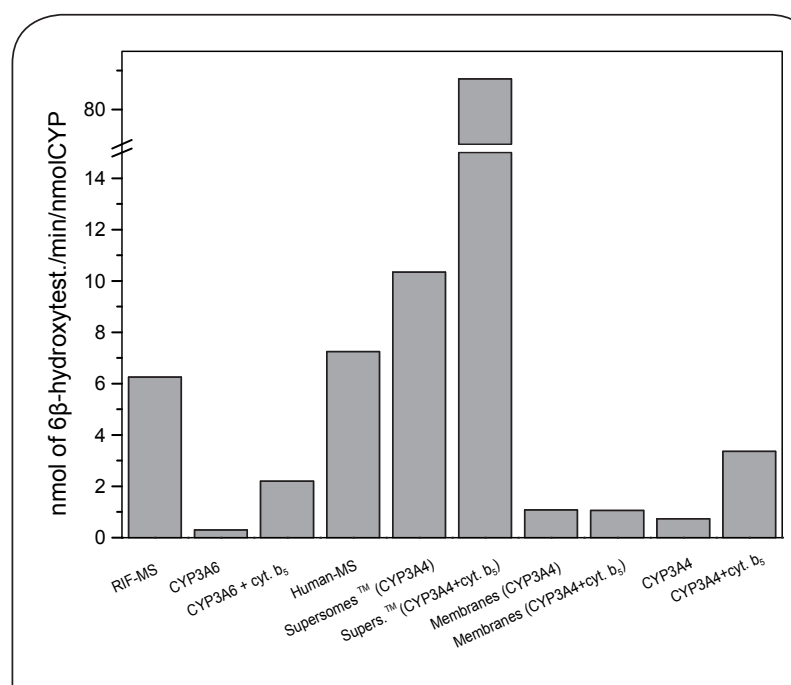

Figure 1. $6 \beta$-Hydroxylation of testosterone by enzymatic systems containing CYP3A4 or CYP3A6.

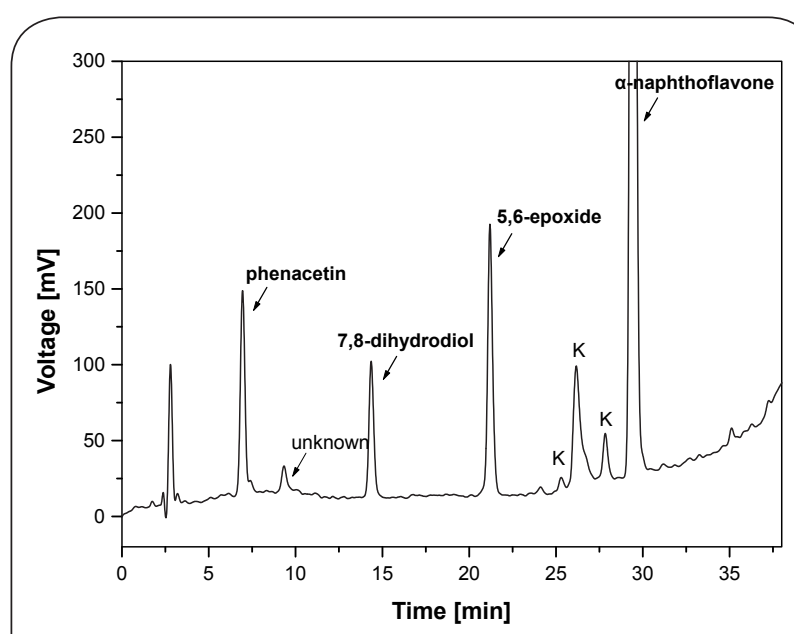

Figure 2. HPLC separation of a-NF metabolites by HPLC formed by incubations of $150 \mu \mathrm{M}$ a-NF with human microsomes (100 pmol (YP) and NADPH-generating system. See Materials and Methods for details. 7,8-dihydrodiol, 5,6-epoxide and unknown metabolite were found. Peaks assigned as $\mathrm{K}$ are present also in control incubations without NADPH-generating system. Phenacetin was used as an internal standard. 


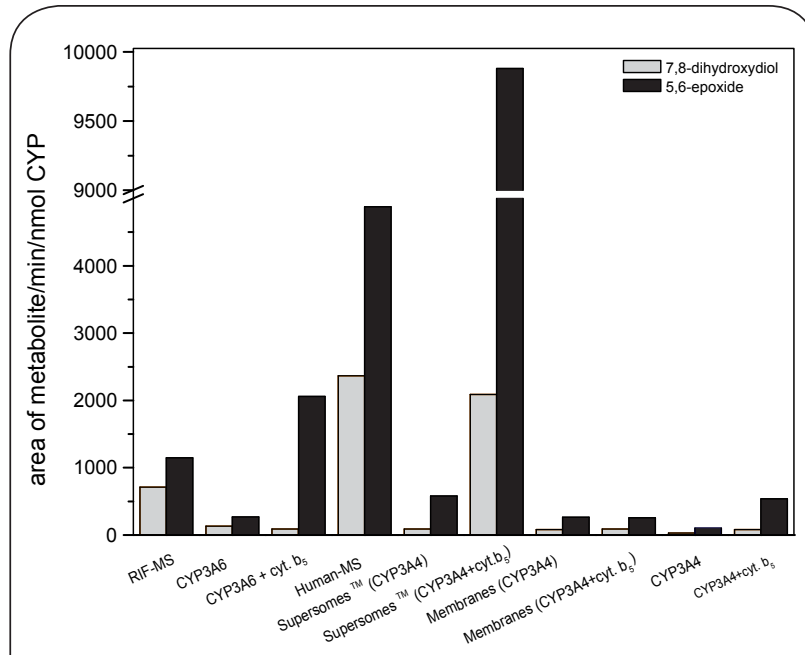

Figure 3. a-NF oxidation by enzymatic systems containing CYP3A4 or CYP3A6.

of CYP3A4 and/or CYP3A6 expressed in membranes of $E$. coli or reconstituted with NADPH:CYP reductase in liposomes to oxidize $\alpha-N F$ were found (Figure 3). Their activity was enhanced by cytochrome $b_{5}$, while cytochrome $b_{5}$ did not influence $\alpha$-NF oxidation by recombinant CYP3A4 of E.coli membranes.

Surprisingly, although 6 $\beta$-hydroxylation of testosterone catalyzed by recombinant human CYP3A4 and NADPH:CYP reductase without cytochrome $b_{5}$ in Supersomes ${ }^{\mathrm{Tw}}$ was very effective, $\alpha$-NF oxidation was low in this system (Figures 1 and 3).

\section{DISCUSSION}

In this report we evaluated CYP3A activity in different enzymatic systems (microsomal and/or reconstituted systems). Under the condition used, the most efficient systems oxidizing testosterone to its $6 \beta$-hydroxylated metabolite and $\alpha$-NF to trans 7,8-dihydrodiol- and 5,6-epoxide were Supersomes $^{\mathrm{Tm}}$ containing human CYP3A4 and cytochrome $b_{5}$. It has been shown that some CYP3A4 activities are dependent on cytochrome $b_{5}$, specific lipid mixtures, cholate, buffer and salt composition (Shimada and Yamazaki 1998, Yamazaki et al., 1996).The results of this study clearly show that the effect of cytochrome $b_{5}$ on CYP3A4-mediated oxidation of testosterone was different from that on oxidation of $\alpha-\mathrm{NF}$. This protein seems to be essential for the effective conversion of $\alpha$-NF to its metabolites. Namely, $6 \beta$-hydroxylation of testosterone catalyzed by recombinant human CYP3A4 and NADPH:CYP reductase in Supersomes ${ }^{\mathrm{Ta}}$ was effective even without cytochrome $b_{5}$. On the contrary $\alpha-\mathrm{NF}$ oxidation by this enzymatic system was negligible without cytochrome $b_{5}$.

The results presented in this study demonstrate the suitability of the supersomal CYP3A4 systems for studies investigating oxidation of testosterone and $\alpha-\mathrm{NF}$ in vitro.
The data indicate that results found in a variety of studies employing different enzymatic systems to evaluate the degree of participation of individual CYP enzymes in xenobiotic metabolism should be carefully interpreted. In order to properly evaluate a role of individual CYPs in metabolism, combination of various in vitro and in vivo approaches should be utilized.

\section{REFERENCES}

Andries, M. J., G. W. Lucier, J. Goldstein, and C. L. Thompson. (1990) Involvement of cytochrome P-450c in alpha-naphthoflavone metabolism by rat liver microsomes. Mol Pharmacol 37: 990-5.

Aoyama, T., S. Yamano, D. J. Waxman, D. P. Lapenson, U. A. Meyer, V. Fischer, R. Tyndale, T. Inaba, W. Kalow, H. V. Gelboin, and et al. (1989) Cytochrome P-450 hPCN3, a novel cytochrome P-450 IIIA gene product that is differentially expressed in adult human liver. cDNA and deduced amino acid sequence and distinct specificities of cDNA-expressed hPCN1 and hPCN3 for the metabolism of steroid hormones and cyclosporine. J Bio/ Chem 264: 10388-95.

Atkins, W. M. (2005) Non-Michaelis-Menten kinetics in cytochrome P450catalyzed reactions. Annu Rev Pharmacol Toxicol 45: 291-310.

Atkins, W. M., R. W. Wang, and A. Y. Lu. (2001) Allosteric behavior in cytochrome p450-dependent in vitro drug-drug interactions: a prospective based on conformational dynamics. Chem Res Toxicol 14: 338-47.

Beaune, P. H., D. R. Umbenhauer, R. W. Bork, R. S. Lloyd, and F. P. Guengerich. (1986) Isolation and sequence determination of a cDNA clone related to human cytochrome P-450 nifedipine oxidase. Proc Natl Acad SCiUSA 83: 8064-8.

Burke, M. D., S. Thompson, C. R. Elcombe, J. Halpert, T. Haaparanta, and R. T. Mayer. (1985) Ethoxy-, pentoxy- and benzyloxyphenoxazones and homologues: a series of substrates to distinguish between different induced cytochromes P-450. Biochem Pharmaco/ 34: 3337-45.

Domanski, T. L., J. Liu, G. R. Harlow, and J. R. Halpert. (1998) Analysis of four residues within substrate recognition site 4 of human cytochrome P450 3A4: role in steroid hydroxylase activity and alpha-naphthoflavone stimulation. Arch Biochem Biophys 350: 223-32.

Ekins, S., D. M. Stresser, and J. A. Williams. (2003) In vitro and pharmacophore insights into CYP3A enzymes. Trends Pharmacol Sci 24: 161-6.

Galetin, A., S. E. Clarke, and J. B. Houston. (2002) Quinidine and haloperidol as modifiers of CYP3A4 activity: multisite kinetic model approach. Drug Metab Dispos 30: 1512-22.

Gonzalez, F. J., and H. V. Gelboin. (1992) Human cytochromes P450: evolution and cDNA-directed expression. Environ Health Perspect 98: 81-5.

Guengerich, F. P. (1999) Cytochrome P-450 3A4: regulation and role in drug metabolism. Annu Rev Pharmacol Toxicol 39: 1-17.

Guryev, O. L., A. A. Gilep, S. A. Usanov, and R. W. Estabrook. (2001) Interaction of apo-cytochrome b5 with cytochromes P4503A4 and P45017A relevance of heme transfer reactions. Biochemistry 40: 5018-31.

Harlow, G. R., and J. R. Halpert. (1998) Analysis of human cytochrome P450 3A4 cooperativity: construction and characterization of a site-directed mutant that displays hyperbolic steroid hydroxylation kinetics. Proc Natl Acad Sci U S A 95: 6636-41.

Haugen, D. A., and M. J. Coon. (1976) Properties of electrophoretically homogeneous phenobarbital-inducible and beta-naphthoflavone-inducible forms of liver microsomal cytochrome P-450. J Biol Chem 251 7929-39.

Hosea, N. A., G. P. Miller, and F. P. Guengerich. (2000) Elucidation of distinct ligand binding sites for cytochrome P450 3A4. Biochemistry 39: 5929-39.

Isin, E. M., and F. P. Guengerich. (2006) Kinetics and thermodynamics of ligand binding by cytochrome P450 3A4. J Biol Chem 281: 9127-36.

Kitada, M., T. Kamataki, K. Itahashi, T. Rikihisa, R. Kato, and Y. Kanakubo. (1985) Purification and properties of cytochrome P-450 from homogenates of human fetal livers. Arch Biochem Biophys 241: 275-80. 
Koley, A. P., J. T. Buters, R. C. Robinson, A. Markowitz, and F. K. Friedman. (1997) Differential mechanisms of cytochrome P450 inhibition and activation by alpha-naphthoflavone. J Bio/ Chem 272: 3149-52.

Kumar, S., D. R. Davydov, and J. R. Halpert. (2005) Role of cytochrome B5 in modulating peroxide-supported cyp3a4 activity: evidence for a conformational transition and cytochrome P450 heterogeneity. Drug Metab Dispos 33: 1131-6.

Omura, T., and R. Sato. (1964) The Carbon Monoxide-Binding Pigment of Liver Microsomes. II. Solubilization, Purification, and Properties. J Biol Chem 239: 2379-85.

Ortiz de Montellano, P. R. (1995) The 1994 Bernard B. Brodie Award Lecture. Structure, mechanism, and inhibition of cytochrome P450. Drug Metab Dispos 23: 1181-7.

Patki, K. C., L. L. Von Moltke, and D. J. Greenblatt. (2003) In vitro metabolism of midazolam, triazolam, nifedipine, and testosterone by human liver microsomes and recombinant cytochromes p450: role of cyp3a4 and cyp3a5. Drug Metab Dispos 31: 938-44.

Reed,J.R., andP.F.Hollenberg. (2003a)Comparison of substratemetabolism by cytochromes P450 2B1, 2B4, and 2B6: relationship of heme spin state, catalysis, and the effects of cytochrome b5. J Inorg Biochem 93: 152-60.

Reed, J. R., and P. F. Hollenberg. (2003b) Examining the mechanism of stimulation of cytochrome P450 by cytochrome b5: the effect of cytochrome b5 on the interaction between cytochrome P450 2B4 and P450 reductase. J Inorg Biochem 97: 265-75.

Shimada, T., and H. Yamazaki. (1998) Cytochrome P450 reconstitution systems. Methods Mol Biol 107: 85-93.

Shimada, T., H. Yamazaki, M. Mimura, Y. Inui, and F. P. Guengerich. (1994) Interindividual variations in human liver cytochrome P-450 enzymes involved in the oxidation of drugs, carcinogens and toxic chemicals: studies with liver microsomes of 30 Japanese and 30 Caucasians. J Pharmacol Exp Ther 270: 414-23.

Shou, M., J. Grogan, J. A. Mancewicz, K. W. Krausz, F. J. Gonzalez, H. V. Gelboin, and K. R. Korzekwa. (1994) Activation of CYP3A4: evidence for the simultaneous binding of two substrates in a cytochrome P450 active site. Biochemistry 33: 6450-5.

Schenkman, J. B., and I. Jansson. (1999) Interactions between cytochrome P450 and cytochrome b5. Drug Metab Rev 31: 351-64.

Stiborova, M., B. Asfaw, E. Frei, H. H. Schmeiser, and M. Wiessler. (1995) Benzenediazonium ion derived from Sudan I forms an 8-(phenylazo) guanine adduct in DNA. Chem Res Toxico/ 8: 489-98.

Stiborova, M., E. Frei, H. H. Schmeiser, M. Wiessler, and J. Hradec. (1990) Mechanism of formation and 32P-postlabeling of DNA adducts derived from peroxidative activation of carcinogenic non-aminoazo dye 1-phenylazo-2-hydroxynaphthalene (Sudan I). Carcinogenesis 11: 1843-8.
Tang, W., and R. A. Stearns. (2001) Heterotropic cooperativity of cytochrome P450 3A4 and potential drug-drug interactions. Curr Drug Metab 2: 185-98.

Thakker, D. R., W. Levin, M. Buening, H. Yagi, R. E. Lehr, A. W. Wood, A. H. Conney, and D. M. Jerina. (1981) Species-specific enhancement by 7,8-benzoflavone of hepatic microsomal metabolism of benzo[e]pyrene 9,10-dihydrodiol to bay-region diol epoxides. Cancer Res 41: 1389-96.

Tsalkova, T. N., N. Y. Davydova, J. R. Halpert, and D. R. Davydov. (2007) Mechanism of interactions of alpha-naphthoflavone with cytochrome P450 3A4 explored with an engineered enzyme bearing a fluorescent probe. Biochemistry 46: 106-19.

Ueng, Y. F., T. Kuwabara, Y. J. Chun, and F. P. Guengerich. (1997) Cooperativity in oxidations catalyzed by cytochrome P450 3A4. Biochemistry 36: $370-81$

Wiechelman, K. J., R. D. Braun, and J. D. Fitzpatrick. (1988) Investigation of the bicinchoninic acid protein assay: identification of the groups responsible for color formation. Anal Biochem 175: 231-7.

Yamada, M., Y. Ohta, G. I. Bachmanova, Y. Nishimoto, A. I. Archakov, and S. Kawato. (1995) Dynamic interactions of rabbit liver cytochromes P450IA2 and P450IIB4 with cytochrome b5 and NADPH-cytochrome P450 reductase in proteoliposomes. Biochemistry 34: 10113-9.

Yamaori, S., H. Yamazaki, A. Suzuki, A. Yamada, H. Tani, T. Kamidate, K. Fujita, and T. Kamataki. (2003) Effects of cytochrome b(5) on drug oxidation activities of human cytochrome P450 (CYP) 3As: similarity of CYP3A5 with CYP3A4 but not CYP3A7. Biochem Pharmacol 66 : 2333-40

Yamazaki, H., W. W. Johnson, Y. F. Ueng, T. Shimada, and F. P. Guengerich. (1996) Lack of electron transfer from cytochrome b5 in stimulation of catalytic activities of cytochrome P450 3A4. Characterization of a reconstituted cytochrome P450 3A4/NADPH-cytochrome P450 reductase system and studies with apo-cytochrome b5. J Biol Chem 271: 27438-44.

Yamazaki, H., M. Nakajima, M. Nakamura, S. Asahi, N. Shimada, E. M. Gillam, F. P. Guengerich, T. Shimada, and T. Yokoi. (1999) Enhancement of cytochrome P-450 3A4 catalytic activities by cytochrome b(5) in bacterial membranes. Drug Metab Dispos 27: 999-1004.

Yamazaki, H., T. Shimada, M. V. Martin, and F. P. Guengerich. (2001) Stimulation of cytochrome $\mathrm{P} 450$ reactions by apo-cytochrome b5: evidence against transfer of heme from cytochrome P450 3A4 to apo-cytochrome b5 or heme oxygenase. J Biol Chem 276: 30885-91.

Yang, C. S., Y. Y. Tu, D. R. Koop, and M. J. Coon. (1985) Metabolism of nitrosamines by purified rabbit liver cytochrome P-450 isozymes. Cancer Res 45: 1140-5.

Yasukochi, Y., J. A. Peterson, and B. S. Masters. (1979) NADPH-cytochrome c $(\mathrm{P}-450)$ reductase. Spectrophotometric and stopped flow kinetic studies on the formation of reduced flavoprotein intermediates. J Biol Chem 254: 7097-104. 